DOI: https://doi.org/10.46630/phm.12.2020.20

Freiderikos E. Valetopoulos ${ }^{1}$

Article de recherche

Université de Poitiers

УДК 811.133.1'243:371.3

Faculté des lettres et des langues

Reçu : le 1/3/2020

Laboratoire de recherche : FoReLLIS (EA 3816) - MSHS (USR 3565)

\title{
L'ENSEIGNEMENT DU VOCABULAIRE EN CLASSE DE FRANÇAIS LANGUE ÉTRANGĖRE : LE CAS DES EMOTIONS
}

Dans le cadre de cet article, nous nous proposons d'étudier l'utilisation du lexique émotionnel par les apprenants de français langue étrangère. Nos remarques se fondent sur l'analyse d'un corpus d'apprenants collecté à l'aide d'un protocole qui s'adresse à des apprenants inscrits au département d'études françaises dans leur pays d'origine, avec un niveau B1/B2 en français langue étrangère. Tout d'abord, nous nous proposons d'examiner quelles sont les habiletés linguistiques que les apprenants doivent développer dans le cadre de l'apprentissage d'une langue étrangère. Ensuite, nous focalisons notre attention sur l'apprentissage du lexique émotionnel et nous présentons les habiletés visées dans le cadre des manuels et les activités utilisées. Enfin, nous analysons une partie du corpus EMO-FLE, celui contenant les productions orales des apprenants sinophones, afin d'observer les moyens qu'ils utilisent pour décrire et exprimer leurs propres émotions.

Mots-clés : Français langue étrangère, émotions, corpus d'apprenants, manuels

\section{Introduction}

Les auteurs du Cadre européen commun de référence pour les langues (dorénavant CECRL 2001 : 86) précisent qu'afin de réaliser des intentions communicatives, les apprenants «mobilisent [d]es aptitudes générales [...] et les combinent à une compétence communicative de type plus spécifiquement linguistique ». Celle-ci comprend les compétences linguistiques, les compétences sociolinguistiques et les compétences pragmatiques. La compétence linguistique est « celle qui a trait aux savoirs et savoir-faire relatifs au lexique, à la phonétique, à la syntaxe et aux autres dimensions du système d'une langue, pris en tant que tel, indépendamment de la valeur sociolinguistique de ses variations et des fonctions pragmatiques de ses

${ }^{1}$ fvaletop@univ-poitiers.fr 
réalisations » (ibidem : 17). A l'intérieur de cette compétence, les auteurs du CECRL distinguent la compétence lexicale, la compétence grammaticale, la compétence sémantique, la compétence phonologique, et enfin la compétence orthographique (ibidem : 87).

Plus précisément, la compétence lexicale, qui sera l'objet de notre analyse dans le cadre de cet article, est la capacité de l'apprenant à utiliser le vocabulaire d'une langue, autrement dit les éléments lexicaux et les éléments grammaticaux (ibidem : 87). D'après les indicateurs du même référentiel, il faut penser à trois paramètres essentiels : la quantité, l'étendue et la maîtrise du vocabulaire (ibidem : 87-89). Pour ce faire, les auteurs partent du principe que les apprenants ont différents moyens d'acquérir ce vocabulaire : la simple exposition, la déduction, la contextualisation, la mémorisation de listes de mots, les dictionnaires etc. (ibidem : 115). Nous pouvons alors nous interroger sur les stratégies que les manuels utilisent pour atteindre ces paramètres. Nous pouvons même nous poser la question de savoir comment les enseignants FLE peuvent les évaluer.

Notre objectif étant de croiser les informations que l'on trouve dans le CECRL avec les informations que l'on obtient à l'aide de l'analyse d'un corpus d'apprenants, nous nous proposons de nous concentrer sur l'analyse de l'utilisation du lexique émotionnel chez les apprenants de français langue étrangère ayant un niveau allant du B1 au B2. Ces deux niveaux sont prioritairement concernés par l'apprentissage du lexique émotionnel (VALETOPOULOS 2014).

\section{La compétence lexicale}

Comme nous l'avons mentionné plus haut, la compétence lexicale est définie comme « la connaissance et la capacité à utiliser le vocabulaire d'une langue » (CECRL 2001 : 87) qui se compose de différents éléments : lexicaux et grammaticaux. Les éléments lexicaux comprennent une longue liste d'éléments tels que les expressions toutes faites, apprises et utilisées comme des ensembles, les proverbes, les archaïsmes, les locutions figées (pour une étude du figement dans les manuels d'enseignement de FLE pour adultes voir par exemple GERBER, LUSTE-CHAÂ 2013), et les mots isolés. À la fin de cette courte présentation, le CECRL propose des échelles pour l'étendue du vocabulaire et la capacité à en maîtriser l'usage. Ces deux notions, étendue et maîtrise, seront définies et mieux explicitées par les auteurs dans la suite du CECRL.

Ainsi, nous pouvons nous référer au chapitre 6, "Quelques options méthodologiques pour l'enseignement »(CECRL 2001 : 103-119), où les 
auteurs reviennent sur la question du développement des compétences linguistiques et plus précisément sur tout ce qui concerne le vocabulaire, la grammaire, la prononciation et l'orthographe. Cette présentation commence par une question pertinente mais qui reste sans réponse : jusqu'où peuton attendre ou exiger des apprenants qu'ils développent leur vocabulaire ? (§6.4.7.1). Les auteurs choisissent en revanche d’énumérer les méthodologies d'enseignement du lexique ou les stratégies que les enseignants peuvent utiliser. Ces approches peuvent être très facilement croisées avec les études réalisées en linguistique appliquée et en didactique des langues, ce que nous tâcherons de faire de manière très brève ci-dessous.

Ainsi, les auteurs du CECRL rappellent que les apprenants peuvent apprendre le lexique par une simple exposition (voir par ailleurs BECK, MCKEOWN et al. 2002) ou par la déduction de l'apprenant (voir aussi LAVOIE 2015, qui étudie trois stratégies pour enseigner le vocabulaire ; cf. SCHMITT 2000, qui développe les stratégies de prédiction) ou l'utilisation d'un dictionnaire. Cela nous rappelle bien évidemment tous les travaux portant sur le principe d'apprendre à apprendre car l'utilisation du dictionnaire demande de la part de l'apprenant une conscience d'apprentissage et surtout des stratégies spécifiques d'utilisation du dictionnaire (voir BOGAARDS 1994 ; GALISSON 1991 ; TREVILLE 2000). L'apprentissage du vocabulaire peut être par ailleurs amélioré quand les nouveaux mots sont présentés en contextes (voir également TREMBLAY 2009 ; TREVILLE, DUQUETTE 1996) ou par des aides visuelles (voir AITCHISON 2012). D'autres moyens portent sur la traduction ou l'approche contrastive à l'aide d'une étude systématique de la distribution des mots en L1 et en L2. Enfin, 1'exploration de champs sémantiques et lexicaux ainsi que l'explicitation de la structure lexicale peuvent également aider à l'apprentissage du lexique.

La deuxième question qui semble être importante concernant l'enseignement du vocabulaire concerne les mots clés qui accompagnent cette compétence, autrement dit la quantité, l'étendue et la maîtrise du vocabulaire (voir \$6.4.7.2). Ces paramètres sont définis par les auteurs du CECRL à l'aide d'une grille de descripteurs, tout en précisant pour autant que (CECRL 2001 : 115) :

« Les utilisateurs du Cadre de référence envisageront et expliciteront selon le cas : - la quantité de vocabulaire que l'apprenant aura besoin de maîtriser ou qu'il devra maîtriser ou dont il devra être outillé (par exemple, le nombre de mots et d'expressions)

- l'étendue de vocabulaire que l'apprenant aura besoin de maîtriser ou qu'il devra maîtriser ou dont il devra être outillé (par exemple, les thèmes, domaines, etc., couverts)

- le type de contrôle du vocabulaire que l'apprenant aura besoin d'exercer ou qu'il devra exercer $[\ldots] »$. 
Quant aux descripteurs nous pouvons lire qu'un apprenant d'un niveau B1, à titre d'exemple, « [p]ossède un vocabulaire suffisant pour s'exprimer à l'aide de périphrases sur la plupart des sujets relatifs à sa vie quotidienne tels que la famille, les loisirs et les centres d'intérêt, le travail, les voyages et l'actualité » alors qu'un apprenant d'un niveau B2 «[p]ossède une bonne gamme de vocabulaire pour les sujets relatifs à son domaine et les sujets les plus généraux. [Il p]eut varier sa formulation pour éviter de répétitions fréquentes, mais des lacunes lexicales peuvent encore provoquer des hésitations et l'usage de périphrases » (ibidem : 88). En ce qui concerne la maîtrise pour les mêmes niveaux, nous pouvons lire qu'un apprenant d'un niveau B1 «[m]ontre une bonne maîtrise du vocabulaire élémentaire mais des erreurs sérieuses se produisent encore quand il s'agit d'exprimer une pensée plus complexe », alors que la maitrise au niveau B2 « est généralement élevée bien que des confusions et le choix de mots incorrects se produisent sans gêner la communication » (ibidem : 89).

Danslevolume complémentaire(CECRLVOLUMECOMPLÉMENTAIRE 2018), les auteurs ajoutent certains descripteurs qui ont pour objectifs d'affiner encore plus ces deux termes qui restent vagues. Ainsi, en ce qui concerne l'étendue du vocabulaire, les auteurs ajoutent pour les apprenants d'un niveau B1 qu'ils ont « une bonne gamme de vocabulaire en rapport avec des sujets familiers et des situations quotidiennes » alors que les B2 peuvent « produire assez systématiquement de nombreux mots adéquats dans la plupart des contextes » et peuvent également « comprendre et utiliser une grande partie du vocabulaire spécialisé de son domaine mais a des difficultés avec la terminologie d'une spécialité différente de la sienne » (CECRL VOLUME COMPLÉMENTAIRE 2018 : 138). Quant à la maitrise du vocabulaire, seul le niveau B1 a été affiné par l'ajout du descripteur : «Utilise une gamme étendue de vocabulaire simple de façon appropriée quand il s'agit de sujets familiers » (CECRL VOLUME COMPLÉMENTAIRE 2018 : 140).

À la suite de cette lecture, nous pouvons nous poser les questions suivantes : Quel est l'impact des manuels sur l'apprentissage du vocabulaire? Quelles sont les stratégies utilisées par les apprenants quand ils ne maîtrisent pas le vocabulaire adéquat? Comment un.e enseignant.e peut-il.elle évaluer la richesse lexicale de ses apprenants ? Comment définissons-nous la richesse / étendue lexicale ? Ce serait difficile de répondre à toutes ces questions dans les limites de notre article. Nous nous proposons de nous limiter aux deux premières questions. 


\section{Le corpus analysé}

Nous avons déjà eu l'occasion de présenter le principe de notre corpus (VALETOPOULOS, RANÇON 2017) qui s'inscrit dans le cadre du projet international Les sentiments à travers les corpus d'apprenants. Ce corpus, qui est évolutif, réunit les productions écrites et orales d'apprenants qui ont différentes langues premières : grec, polonais, chinois etc. Pour les besoins de cet article, nous nous concentrerons sur les productions orales qui ont été réalisées sous format d'entretien. Ces entretiens ont eu pour objectif de décrire oralement en français quatre images. Ces images pouvaient faire réagir à la situation proposée, pouvaient permettre de décrire ses propres émotions, pouvaient rendre compte des émotions d'une autre personne et enfin, avaient pour ambition de provoquer des émotions. Les entretiens ont été enregistrés (corpus EMO-FLE). L'objectif de cette recherche était d'amener les apprenants à décrire et commenter ces images. La description permettait de poser un cadre thématique dans lequel le contour émotionnel les invitait à parler éventuellement d'eux-mêmes. La question Quelles sont les émotions que vous éprouvez à la vue de cette image? assurait la présence d'un discours émotionnel. Ainsi, les apprenants faisaient référence à des thématiques plus personnelles, communes ou différentes, selon la communauté linguistique à laquelle ils appartenaient. Pour les besoins de cet article, nous utiliserons les données qui proviennent du sous-corpus d'apprenants sinophones. Comme pour les autres communautés linguistiques, nous avons réalisé cet entretien avec vingt apprenants, étant inscrits au département d'études françaises dans leur pays d'origine et ayant un niveau B1/B2 en français langue étrangère.

\subsection{Le choix du champ sémantique des émotions}

Le choix du champ sémantique des émotions est motivé par différentes raisons. Nous pouvons dire que les émotions présentent un double intérêt pour la didactique des langues car soit on peut les étudier en tant que facteur important dans l'acquisition des langues (PUOZZO CAPRON, PICCARDO 2013) soit en tant qu'observable linguistique qui peut ou qui doit être enseigné. En ce qui concerne le premier aspect, nous pouvons juste souligner ce qui a déjà été souligné par les deux auteurs, PUOZZO CAPRON et PICCARDO (2013), dans leur introduction :

"À l'école, l'une des finalités est l'acquisition progressive d'une autonomie cognitive qui permet à l'élève d'autoréguler ses apprentissages dans les différentes disciplines. Le substrat de ces apprentissages peut aussi être l'émotion qu'il faut apprendre progressivement à gérer. L'autonomie émotionnelle, dans le sens d'être capable de s'autoréguler émotionnellement, est une finalité transversale souvent perdue de vue au profit des contenus disciplinaires. » 
Bien évidemment, cela nous fait penser à tous les travaux portant sur l'affectivité en tant que filtre ayant un impact sur la motivation et la confiance en soi (KRASHEN 1981 ; voir également la discussion sur les stratégies affectives, CYR 1998).

Mais les émotions en tant qu'élément observable et enseignable a commencé également à intéresser les linguistes et les didacticiens depuis quelques années. Nous pouvons ainsi lire chez GROSSMAN et BOCH (2003 : 121):

« [d]'une part, ce champ présente un intérêt humain évident, qui trouve matière à s'exprimer à travers l'expérience personnelle des jeunes scripteurs mais aussi dans les récits et les fictions de toutes sortes ; écrire un récit d'expérience ou fictionnel suppose que le scripteur soit en mesure de traduire un point de vue, ce qui passe généralement par la traduction de ses sentiments personnels ou de ceux qu'il fait éprouver aux personnages qu'il met en scène. D'autre part, ce lexique est abstrait, difficile par sa richesse conceptuelle, assez peu mobilisé dans sa diversité par les élèves dans leurs productions ».

Nous devons aussi rappeler les travaux dans le cadre du bilinguisme qui montrent que l'utilisation du champ sémantique des émotions par les locuteurs bilingues semble être soumise à différentes conditions (DEWAELE, PAVLENKO 2002 : 298) :

« $[\ldots]$ the two languages/cultures in consideration, the level of language proficiency, introversion/extraversion, gender of the speakers (and possibly their interlocutors), context of the interaction, and the type of linguistic material in question may influence the range and the frequency of use of emotion words in interlanguage. »

Pour résumer, nous pouvons tout simplement insister sur la complexité que l'on observe au niveau syntaxique et sémantique. Ainsi, les prédicats qui expriment une émotion présentent un intérêt singulier :

- au niveau syntaxique, les apprenants doivent les construire avec la préposition de ou la complétive que + subjonctif,

- au niveau lexical, ils doivent conceptualiser les différences qui existent entre différents prédicats tels que l'intensité (surprise vs. stupéfaction), le niveau de langue (peur vs. pétoche), le sens (peur vs. crainte),

- au niveau phraséologique, ils doivent apprendre la combinatoire lexical (se mettre en colère $+*$ en surprise), ou enfin

- au niveau de l'intonation, les apprenants doivent être capables de reconnaitre l'intonation utilisée dans une langue étrangère pour exprimer la joie ou la colère. 
En ayant toutes ces observations en tête, nous nous sommes donc intéressé à deux autres aspects qui ont un rapport avec l'enseignement du vocabulaire : a. quelles sont les habiletés développées dans le cadre des manuels, et b. que pouvons-nous observer à travers le corpus d'apprenants ?

\subsection{Les habiletés linguistiques visées par les manuels}

Quand un apprenant doit exprimer ses émotions, il peut le faire par le biais de différents moyens d'expression. Ainsi, nous considérons, comme nous l'avons déjà suggéré (VALETOPOULOS 2016), que les apprenants doivent tout d'abord être capables de réagir afin d'exprimer une émotion. Ainsi, l'apprenant joue le rôle de l'émetteur : il réagit à un stimulus et émet un discours émotionnel expressif. C'est ce que l'on peut voir dans le cadre de différentes activités comme par exemple dans l'activité suivante permettant aux apprenants d'associer une émotion à une intonation :

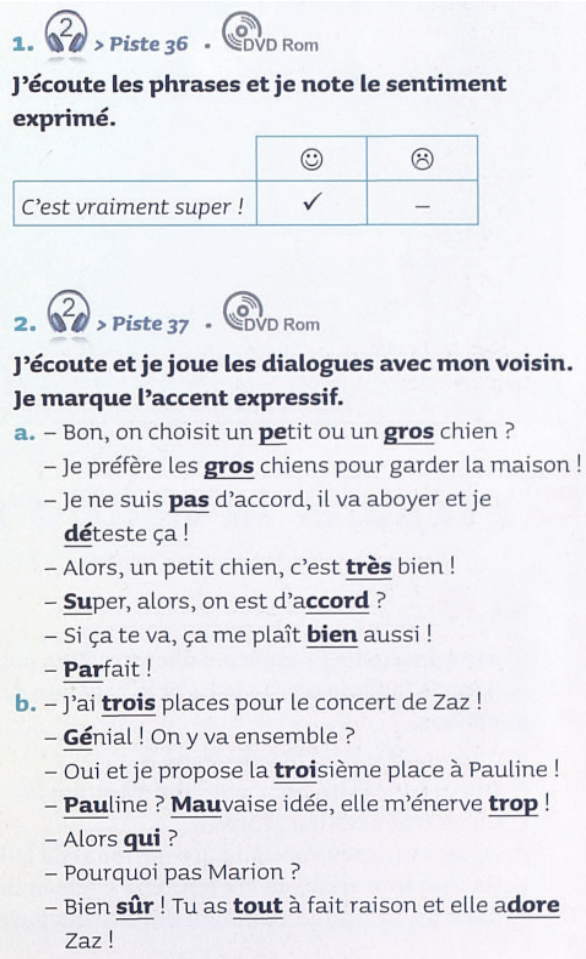

Image 1. Agenda 2 (BAGLIETO, GIRARDEAU et al. 2011), p. 87

L'apprenant doit être capable de décrire ses émotions étant lui-même le siège psychologique : il dit ce qu'il ressent ou il dit ce qu'il ressent adossant le 
rôle d'un personnage. L'apprenant émet un discours émotionnel descriptif. La différence par rapport à la première habileté se situe dans le fait que l'apprenant conceptualise ses émotions et donne un nom à ces émotions. Par exemple,

B. Expliquez à vos camarades comment vous vous sentez en classe de langue.

- Moi, j'aime beaucoup faire des rédactions i par contre, je me sens ridicule quand je parle en français.

Image 2. Version Originale 2 (DENYER, GARMENDIA et al. 2014), p. 15

(4)

J'écris un courrier à un ami pour exprimer mes sentiments (joie, tristesse, surprise ou déception).

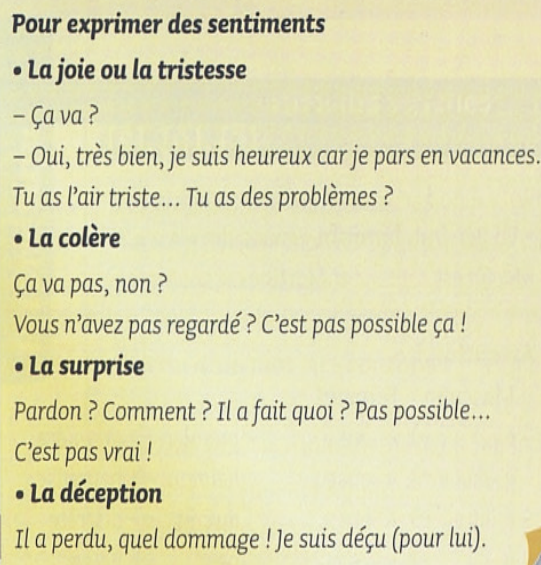

Image 3. Agenda 2 (BAGLIETO, GIRARDEAU et al. 2011), p. 69

\section{(4)) EX. 4. Écoutez les extraits musicaux suivants et écrivez les sentiments ou émotions qu'ils vous inspirent.}

Image 4. Entre nous 3 (AVANZI, MALOREY et al. 2016a), p. 39

L'apprenant doit être également capable de rapporter les émotions de quelqu'un d'autre. Il fonctionne en tant que médiateur pour parler des émotions des autres. Par exemple, nous pouvons lire dans les deux activités suivantes : je lis la lettre et je dis comment se sent Andrée Naline (Image 5) ou Lisez les lettres. Quel(s) sentiment(s) expriment-elles? 


\section{EXPRIMER DES SENTIMENTS}

\section{(1)}

Je lis la lettre et je dis comment se sent Andrée Naline.

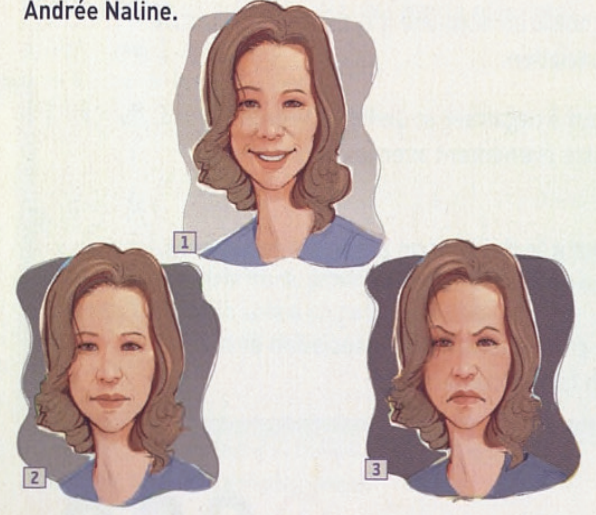

2

Je lis encore la lettre et j'écris les expressions qui expriment des sentiments.

(3) 2 > Piste 8 . ODV Rom

J'écoute le document et j'associe les personnes aux sentiments.
a. Personne 1
b. Personne 2
c. Personne 3

1.
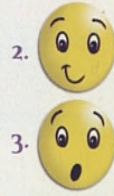

Image 5. Agenda 2 (BAGLIETO, GIRARDEAU et al. 2011), p. 68

B. Lisez les lettres. Quel(s) sentiment(s) expriment-elles ? Connaissez-vous les auteures et leurs destinataires? Faites des recherches si nécessaire.

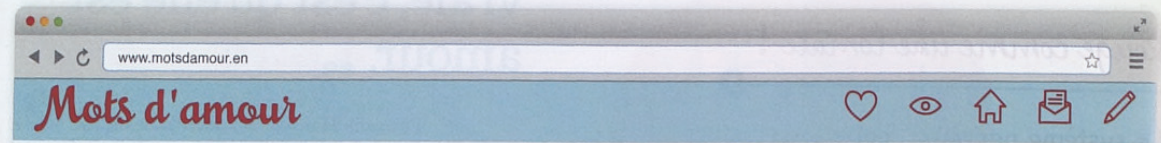

Spécial Saint- Qualentin
Cette année, épatez votre partenaire en lui écrivant la plus belle des lettres d'amour ! Nous avons sélectionné pour vous deux magnifiques
lettres, l'une d'Édith Piaf adressée à Marcel Cerdan, et l'autre de Juliette Drouet à Victor Hugo. Inspirez-vous!

Mon adoré.

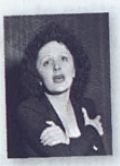

J'ai tant de choses à te dire et puis tout se bouscule. Et je me rends compte à chaque fois que je termine une lettre, que javais encore mille trued à écrire et que cést encore une fois trop tard. La seule phrase que je rioublie jamais c'est que je t'aime de plus en plus et que je suls complètement folle de toi. C'est vaui. mon adoré. je m' habitue de moins en moins à nos séparations et mon cocut se déchire à chaque fois un peu plus. Je t'aime si profondèment. di fort dans mot. je suis imprégnée de toi et ríai quiune seule idée. te nendre heureux. Je serais capable de tout poun ton bonheur. Si tu savais les idées qui me traversent la tête. fai tant peur que tu aies de la peine à cause de mot. je ne veux jamais être une entrave à ton caeur. (.J Je me jette dans tes bras que fadore. je t'appartiens, tout petit adoré que faime. Serre-moi fort contre ton coeur. empêche-moi de nespirer. Edith limon bien-aimé,

Te t'ui quitté mon bien-aimé. Que le souvenir de notre amour te suive et te console pendant notre séparation. Si lu savais combien je l'aime, combien lu es nécessaire à ma vie, Iu noserais pas tabsenter un seul moment, tu resterdis toyjours auprès de moi, ton caur contre mon caur, ton âme contre mon âme. $\mathscr{H}$ est onze heures du soir. Te ne l'ai pas vu, je l'uttends avec bien de l'impatience, je l'altends coyjours. $\mathscr{T}$ me semble qu'ily a un siècle queje ne l'ai, vu, que je n'ai contemplé tes lraits, que je ne me suis eniorée de ton regard.(...). Ifon Tictor, pardonne-moi toutes mes folies.

Guliette

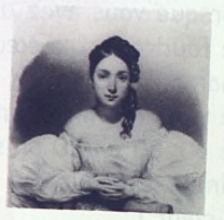

Image 6. Entre nous 4 (AVANZI, MALOREY et al. 2016b), p. 68 
Enfin, l'apprenant doit être capable de susciter des émotions. L'apprenant adosse le rôle du créateur d'émotion par les mots, par les gestes, par les images, par les représentations. Dans les différents manuels que nous avons pu étudier, nous n'avons pas relevé des activités qui ont pour objectif cette habileté. Il s'agit sans doute d'une compétence développée à l'aide d'autres activités annexes, telles que la production d'un texte ou la rédaction d'une lettre qui pourrait évoquer des émotions chez un lecteur imaginaire.

\subsection{Le corpus d'apprenants}

À la lumière de ces habiletés visées par les manuels et par le CECRL (voir chapitre 2), nous nous intéressons maintenant aux productions d'un groupe d'apprenants sinophones qui ont décrit les images utilisées dans le cadre de l'expérimentation. Examinons les occurrences relevées dans le cadre de la première image qui a pour vocation de créer un sentiment positif.

(1) oh euh je me ++ je me sens ah s- euh + euh ça me rend- ça me rend euh ça m- mh euh j- ++ euh ça me rend être à- à l'aise parce que je j-j'adore la plage et la mer

(2) c'est une image tranquille + et + je me sens très + mh très calme et + c'est- peut-être c'est dans- dans- la fille est dans- dans ses vacances comme ça + oui c'est

(3) + je- je sentis très à l'aise car euh c'est pour moi une image très + très beau + et tr- très belle et $+\mathrm{s}$ - je suis bien-être en voyant cela- cel- cette image

(4) ah perroquet d'accord et + mh je pense c'est une image très + mh agréable

(5) hum je me sens + je me ressens ++ s- soulagée

(6) je pense que mh je me sens très hum + comment on dit en français relax $\{$ anglais\}

Nous pouvons constater que les apprenants utilisent un lexique émotionnel plutôt approximatif comme par exemple être à l'aise ou *être bien-être. Nous n'avons relevé que deux occurrences comme se sentir calme et se sentir soulagée qui pourraient correspondre plutôt au contexte. Il faut souligner par ailleurs qu'ils choisissent de décrire l'image (tranquille, beau, agréable) afin d'exprimer indirectement leurs propres émotions.

C'est par ailleurs ce que nous pouvons observer dans le cadre des réponses qui suivent pour les photos exprimant une émotion plutôt négative telle que la tristesse ou la colère.

(7) $++m h+$ je euh mh je pense euh c'est euh cette fille est très + euh très marquante + inoubliable 
$(8)+$ et ça me donne un peu pression + la pression + oui mais ça me sentit pas très confortable

(9) $\mathrm{mh}+$ les gens sont en colère et ils euh expriment leurs émotions par une manifestation

(10) je pense c'est un peu un peu triste + mais je ne sais pas pourquoi mais je vu quelques + je pense euh elle est triste + oui elle est en colère

$(11)+$ c'est une image bizarre

Nous pouvons constater les mêmes stratégies que pour l'émotion positive (joie) même si les occurrences sont moins nombreuses. Les apprenants décrivent soit l'image par des adjectifs tels que marquant ou bizarre soit les émotions ressenties par le personnage de l'image, par exemple en colère ou triste. Nous pouvons souligner deux occurrences qui se réfèrent à la situation émotionnelle des apprenants : pression et pas confortable.

L'analyse des occurrences concernant la photo qui montre un garçon qui pleure (12-14) et la photo qui évoque un sentiment triste (15-18) témoigne ce manque au niveau du lexique émotionnel. Les apprenants utilisent le terme triste pour exprimer leur propre émotion ainsi que les prédicats génériques touché, ému, inconfortable, bizarre.

(12) $\mathrm{mh}+$ je me sens un peu + triste + oui quand je XXX

(13) touchée

(14) quand je vu cette photo je- je me sens un peu bizarre +

$(15)+$ et c- c'est un peu $+m h+$ inconfortable +

(16) oui ça me ressent- ça me sent euh triste

(17) euh ça me touche + il y a beaucoup de émo- émotions + je pense je- je pense j'ai déjà vu le

(18) euh c'est euh + émue

Passons maintenant à une discussion des résultats tout en les croisant avec nos observations d'ordre théorique.

\section{Discussion}

Les auteurs du CECRL proposent différents indicateurs afin d'évaluer la progression de la compétence lexicale. Ces indicateurs sont accompagnés d'un certain nombre de stratégies d'enseignement du lexique, présentés plutôt à titre indicatif. Dans notre approche, nous avons souhaité formaliser les différentes utilisations du lexique émotionnel par les apprenants. Nous avons ainsi insisté 
sur le fait que les apprenants doivent être capables tout d'abord d'exprimer leurs émotions en réagissant à un stimulus, ensuite ils doivent avoir un lexique suffisant leur permettant de décrire leurs émotions ainsi que les émotions des autres, et enfin pouvoir provoquer des émotions chez leurs interlocuteurs à travers différentes stratégies. Ces différentes approches semblent être reprises dans le cadre de différents manuels qui, depuis quelques années, tentent de varier et d'enrichir leurs activités. Les auteurs ont conscience de la complexité de la tâche et proposent une batterie d'activités permettant ainsi aux apprenants de découvrir différents aspects du lexique émotionnel.

La question que l'on doit se poser maintenant est de savoir si cette richesse au niveau de l'enseignement se reflète dans les productions orales des apprenants. Nous avons ainsi mis en place une expérimentation permettant aux apprenants de produire un discours émotionnel. Une première analyse du lexique rencontré dans les productions orales montre que les apprenants ont du mal à mobiliser un lexique diversifié. Nous arrivons alors à des résultats équivalents aux résultats déjà observés dans le cadre des apprenants hellénophones : lexique plutôt limité, des mots à charge émotionnelle pour décrire la situation ou des mots très génériques pour décrire leurs propres émotions. Autrement dit, les apprenants ne semblent pas disposer d'un lexique suffisant leur permettant de décrire leurs émotions ou les émotions des autres. Mais rappelons ce que nous avons déjà souligné (VALETOPOULOS, LAY 2015) :

[...] l'observation de la diversité du lexique mobilisé n'est pas forcément un bon indicateur : les habiletés linguistiques sont aussi de combinaison. Au-delà de la présence/absence d'un vocabulaire notionnel maîtrisé, il faut évaluer la combinatoire des noms et des adjectifs, celle de la modalisation verbale ou adverbiale, celle de l'utilisation agile des régularités structurelles. L'évaluation d'une production orale suppose aussi la prise en compte de la déixis ou encore du mimo-gestuel, particulièrement important dans la manifestation des émotions. Et cette compétence-là s'acquiert autrement que par l'apprentissage de stratégies strictement linguistiques : il s'agit de mobiliser ce qui, dans la parole, vient du corps (voix, regards, postures) et ne peut se faire que dans un échange, une circulation entre les interlocuteurs.

Ainsi, nous devons revenir sur notre question initiale : peut-on évaluer les trois paramètres, quantité, étendue et maîtrise du vocabulaire, quand on parle du vocabulaire émotionnel ? La réponse dépend de ce que l'on souhaite évaluer. S'il faut évaluer uniquement la connaissance du lexique émotionnel, on peut en effet se concentrer sur le nombre de mots appartenant à cette classe sémantique. Mais si l'on souhaite évaluer la compétence des apprenants de comprendre une émotion, d'exprimer une émotion ou de provoquer une émotion, nous devons aller au-delà de cette simple connaissance du lexique. 


\section{Bibliographie}

AITCHISON 2012 : AITCHISON, Jean. Words in the Mind: An Introduction to the Mental Lexicon. Oxford : John Wiley \& Sons, 2012.

AVANZI, MALOREY et al. 2016a : AVANZI, Audrey et Céline MALOREY, Neige PRUVOST, Caroline VENAILLE, Thomas GEERAERT, Grégory MIRAS, Sylvie POISSON-QUINTON. Entre nous 3, Méthode de français. Paris : Maison des langues, 2016.

AVANZI, MALOREY et al. 2016b : AVANZI, Audrey et Céline MALOREY, Lisa PRUNIERES, Neige PRUVOST, Charlotte JADE, Grégory MIRAS, Sylvie POISSON-QUINTON. Entre nous 4, Méthode de français. Paris : Maison des langues, 2016.

BAGLIETO, GIRARDEAU et al. 2011 : BAGLIETO, David et Bruno GIRARDEAU, Mickaël MAGNE, Marion MISTICHELLI. Agenda 2, Méthode de français. Paris : Hachette.

BECK, MCKEOWN et al. 2002 : BECK, Isabel and Margaret MCKEOWN, Linda KUCAN. Bringing Words to Life: Robust Vocabulary Instruction. New York / London: The Guilford Press, 2002.

BOGAARDS 1994 : BOGAARDS, Paul. Le vocabulaire dans l'apprentissage des langues étrangères. Paris : Hatier, 1994.

CECRL VOLUME COMPLEMENTAIRE 2018 : Conseil de l'Europe. Un cadre européen commun de référence pour les langues : apprendre, enseigner, évaluer. Volume complémentaire avec de nouveaux descripteurs. 2018. <https://rm.coe.int/cecr-volume-complementaireavec-de-nouveaux-descripteurs/16807875d5>. 17.03.2020.

CECRL 2001 : Conseil de l'Europe. Un cadre européen commun de référence pour les langues : apprendre, enseigner, évaluer. Paris : Didier, 2001.

DENYER, GARMENDIA et al. 2014 : DENYER Monique et Agustin GARMENDIA, Corinne ROYER, Marie-Laure LIONS-OLIVIERI. Version originale 2, Méthode de français. Paris : Maison des langues, 2014.

CYR 1998 : CYR, Paul. Les Stratégies d'apprentissage. Paris : CLE International, 1998.

DEWAELE, PAVLENKO 2002 : DEWAELE, Jean-Marc et Aneta PAVLENKO. « Emotion Vocabulary in Interlanguage ». Language Learning 52/2 (2002) : 263-322.

GALISSON 1991 : GALISSON, Robert. De la langue à la culture par les mots. Paris : CLE international, 1991.

GERBER, LUSTE-CHAÂ 2013 : GERBER, Nathalie et Olha LUSTECHAÂ. «Traitement du figement dans les manuels d'enseignement / apprentissage de FLE pour adultes ». Pratiques 159-160 (2013) : 228246. 
GROSSMANN, BOCH 2003 : GROSSMANN, Francis et Françoise BOCH. « Production de textes et apprentissage lexical : l'exemple du lexique de l'émotion et des sentiments ». Repères 28 (2003) : 117-135.

KRASHEN 1985 : KRASHEN, Stephen. The Input Hypothesis: Issues and implications. London : Longman, 1985.

LAVOIE 2015 : LAVOIE, Constance. « Trois stratégies efficaces pour enseigner le vocabulaire : une expérience en contexte scolaire innu ». Revue canadienne de linguistique appliquée 18/1 (2015) : 1-20.

PUOZZO CAPRON, PICCARDO 2013 : PUOZZO CAPRON, Isabelle et Enrica PICCARDO (dir.), L'émotion et l'apprentissage des langues, LIDIL 48. Grenoble : Ellug, 2013.

SCHMITT 2000 : SCHMITT, Norbert. Vocabulary in language teaching. Cambridge: Cambridge University Press, 2000.

TREMBLAY 2009 : TREMBLAY, Ophélie. Création d'une ontologie des connaissances métalexicales pour l'élaboration d'un module de cours en didactique du lexique destiné aux futurs maîtres au primaire en français langue maternelle. Thèse de doctorat inédite. Université de Montréal, Montréal, Canada. 2009.

TREVILLE 2000 : TREVILLE, Marie-Claude. Vocabulaire et apprentissage d'une langue seconde : recherches et théorie. Montréal : Éditions Logiques, 2000.

TREVILlE, DUQUETTE 1996 : TREVILLE, Marie-Claude et Lise DUQUETTE. Enseigner le vocabulaire en classe de langue. Vanves : Hachette, 1996.

VALETOPOULOS 2014 : VALETOPOULOS, Freiderikos. « Les sentiments dans les manuels de FLE ». Inspiracja motywacja sukces : rola materiałów dydaktycznych i form prazy na lekcji języka obcego (2014) : 47-60.

VALETOPOULOS 2016 : VALETOPOULOS, Freiderikos. « Quand les apprenants parlent de leurs émotions : une étude des adjectifs subjectifs à travers les corpus d'apprenants ». Les émotions et les valeurs dans la communication I (2016) : 55-66.

VALETOPOULOS, LAY 2015 : VALETOPOULOS, Freiderikos et MarieHélène LAY. " Exprimer ses émotions à l'oral : étude d'un corpus d'apprenants ». Regards sur l'oral et l'écrit (2015) : 218-223.

VALETOPOULOS, RANÇON 2017 : VALETOPOULOS, Freiderikos et Julie RANÇON, "Thématiques dans le discours émotionnel d'apprenants allophones. Quelles perspectives pour l'enseignement-apprentissage des langues étrangères ? », Philologia Mediana 9 (2017) : 63-77. 
Фредерикос Е. Валетопулос

\section{ВОКАБУЛАР У НАСТАВИ ФРАНЦУСКОГ КАО СТРАНОГ ЈЕЗИКА: ПРИМЕР ЕМОЦИЈА}

У овом раду разматрамо емоционалну лексику којом се служе студенти француског као страног језика. Наша запажања темеље се на анализи корпуса састављеног на основу тестирања студената на нивоима Б1/Б2, уписаних на студије француског језика у матичној земљи. Пре свега желимо да испитамо које су то језичке вештине које испитаници морају развити током учења страног језика. Потом усмеравамо пажњу на усвајање емоционалне лексике и приказујемо вештине које су предвиђене у анализираним уџбеницима, као и активности које се користе за стицање датих вештина. Напослетку, анализирамо део EMO-FLE корпуса који садржи усмену продукцију синофоних студената како бисмо утврдили којим се средствима користе не би ли описали и изразили своје емоције.

Кључне речи: француски као страни језик, емоције, корпус испитаника, уџбеници 